\title{
The Genomic Structure of a Mouse Seminal Vesicle Autoantigen ${ }^{1}$
}

\author{
Lung-Chih Yu,* Ya-Ling Hsiao, $†$ Yun-Hsin Yang, † Marie Lin,* and Yee-Hsiung Chen† ${ }^{* 2}$ \\ *Department of Medical Research, Mackay Memorial Hospital, †I nstitute of Biochemical Science, College of Science, \\ National Taiwan University, and ¥I nstitute of Biological Chemistry, Academia Sinica, Taipei, Tai wan
}

The genomic structure of an androgen-stimulated mouse seminal vesicle autoantigen was determined. Analysis of the nucleotide sequence established 2135 bp of the $5^{\prime}$-flanking region, four exons of 123, 136, 112, $227 \mathrm{bp}$, three introns of 1555, 1931, $316 \mathrm{bp}$, and $185 \mathrm{bp}$ of the 3 '-flanking region of this gene. Ten DNA segments, five in the $5^{\prime}$-flanking region, two in the first intron, and three in the second intron were identified to have more than $50 \%$ homology with the consensus sequence of the androgen response element (ARE). Two sets of adjacent DNA segments, one including -213 to -199 bp and -124 to -110 bp in the $5^{\prime}$-flanking region and the other including 2532 to 2546 bp and $\mathbf{2 5 8 2}$ to $\mathbf{2 5 9 6}$ bp in the second intron, are noticeable for their high degree of homology with ARE. ๑1997 Academic Press

The seminal vesicles of adult male mammals secrete a group of products that constitute a major portion of seminal plasma. The primary structure for some of the major protein components in mouse and rat seminal vesicle secretions have been established (1-3), but still no significant clues to their function could be inferred from their protein structures, despite that their gene expression is shown to be androgen-dependent. Recently, we have identified an androgen-stimulated autoantigen (SVA) in mouse seminal vesicle (4). It is a zinc-binding $19-k D a$ glycoprotein with a core protein consisting of 131 amino acid residues $(4,5)$.

Androgenic action is considered to be mediated through the interaction between a hormonal receptor

\footnotetext{
${ }^{1}$ The nucleotide sequence reported in this paper has been registrated in the GenBank/EMBL Data Bank (Accession number: L44117).

${ }^{2}$ To whom correspondence should be addressed: Institute of Biochemical Science, College of Science, National Taiwan University. P.O. Box 23-106, Taipei, Taiwan. Fax: 886-2-363-5038. E-mail: BC304@gate.Sinica.edw.tw.
}

complex and the androgen response element (ARE) in the responsive gene (6). In spite of the presence of androgen receptor in many sexual organs, the expression of SVA occurs exclusively in seminal vesicle (4). Thus, establishing the genomic structure of SVA becomes a prerequisite toward a better understanding of how the androgen regulation for the transcription of this gene in seminal vesicle. This work was carried out in this direction. Here, we report the genomic structure of the SVA gene and the identification of ARE-like sequences in the SVA gene.

\section{METHODS}

\section{Genomic Cloning and Sequencing of the SVA Gene}

A genomic library of mouse B6/CBAF 1 J spleen DNA in lambda FIX II vector (Stratagene, La J olla, CA, U.S.A.) was screened. The hybridization probe was randomly primed from the full length CDNA of SVA (4). A positive lambda phage clone containing a $18 \mathrm{~kb}$ genomic DNA insert was isolated and denoted as 11S. The $18 \mathrm{~kb}$ DNA insert was digested with Sacl restriction enzyme and then analyzed by southern blotting technique using the same random-primed probe. Two DNA fragments of 4.2 and $5.5 \mathrm{~kb}$ were hybridized with the probe. The $4.2 \mathrm{~kb}$ fragment was further cleavaged into 2.7 and 1.5 $\mathrm{kb}$ fragments by EcoRI digestion. Only the $2.7 \mathrm{~kb}$ fragment bound to the hybridization probe in the southern blotting analysis. The 2.7 $\mathrm{kb}$ and $5.5 \mathrm{~kb}$ fragments were subcloned in pGEM 7Zf(-) vector (Promega, Madison, WI, U.S.A.).

DNA sequences were determined by the dideoxynucleotide chain termination method using an AmpliCycle Sequencing Kit (Perkin Elmer, Foster City, CA, U.S.A.).

\section{Primer Extension Analysis}

A synthetic oligonucleotide corresponding to the antisense sequence in the first exon (nucleotides +91 to +114 ) of the SVA gene was used in primer extension analysis. Total RNA from seminal vesicles of adult mice ( 10 weeks old) was isolated by guanidinium thiocyanate/cesium chloride cushion method (7). Twelve ng of the primer was hybridized to $6 \mu \mathrm{g}$ of total RNA in reverse transcription buffer $(50 \mathrm{mM} \mathrm{NaCl}, 34 \mathrm{mM}$ Tris- $\mathrm{HCl}$ and $5 \mathrm{mM}$ DTT, $\mathrm{pH}$ 8.3) at $85^{\circ} \mathrm{C}$ for $10 \mathrm{~min}$, followed by $45^{\circ} \mathrm{C}$ for $12 \mathrm{~h}$. dGTP, dTTP and dCTP each at a final concentration of $0.25 \mathrm{mM}$ and $8 \mu \mathrm{Ci}$ of $\left[\alpha-{ }^{32} \mathrm{P}\right] \mathrm{dATP}$ (2.7 pmole) were added to the annealing mixture and incubated 


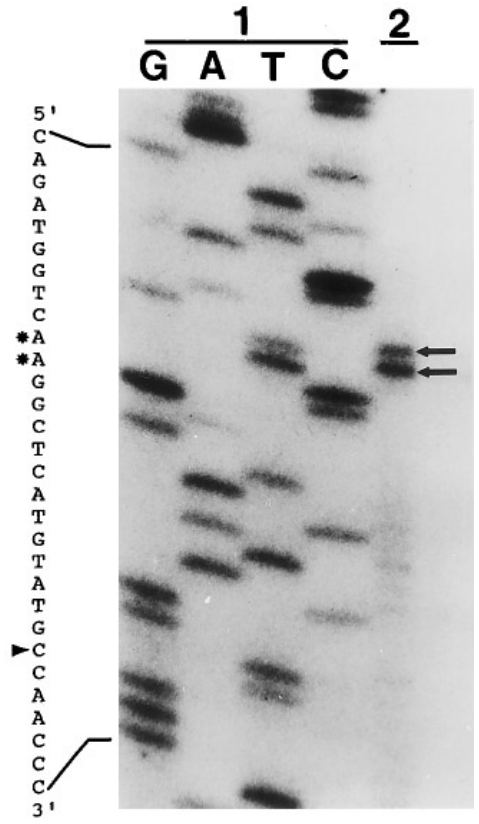

FIG. 1. Primer extention analysis of the transcription initiation sites of the SVA gene. The two major products indicated by arrows in lane 2 were determined from primer extension analysis using total RNA isolated from seminal vesicle of adult mice as template and oligonucleotide corresponding to the antisense sequence in the first exon as primer (see Methods for details). The sequence shown on the left side was read from the sequencing ladders (columns $G, A, T$, and $C$ in lanes 1 ) of the 115 lambda DNA using the same oligonucleotide as primer. The nucleotides corresponding to the extented products, at positions -28 and -27 upstream from the ATG start codon, are marked by "*”.

with 3 units of reverse transcriptase from Avian Myeloblastosis Virus (Promega) in a final volume of $6 \mu \mathrm{l}$ at $42^{\circ} \mathrm{C}$ for $15 \mathrm{~min}$. One $\mu$ l of dNTP mixture ( $2 \mathrm{mM}$ each) was then added to the reaction solution and chased for another $15 \mathrm{~min}$ at $42^{\circ} \mathrm{C}$. The reaction was stopped by adding $5 \mu \mathrm{l}$ of $98 \%$ formamide containing 10 mM EDTA, $0.3 \%$ xylene cyanole and $0.3 \%$ bromophenol blue.

The lambda DNA from $11 \mathrm{~S}$ clone was served as template for dideoxynucleotide sequencing using the same oligonucleotide primer. The sequencing reaction products were run parallel with the primer-extended fragments on a $6 \%$ poly-acrylamide/urea sequencing gel.

\section{RESULTS AND DISCUSSION}

The transcription initiation site of the SVA gene was determined by primer extension analysis. The two major products were corresponding to the nucleotides at 27 and 28 bp upstream from the ATG start codon (Fig. $1)$, revealing the presence of two successive transcription initiation sites in the SVA gene.

Through subcloning and sequencing of the 2.7 and $5.5 \mathrm{~kb}$ DNA fragment (see Methods), the genomic structure of SVA was established. We determined the complete sequence of $2720 \mathrm{bp}$ from the $2.7 \mathrm{~kb}$ fragment and the partial sequence of $4000 \mathrm{bp}$ from the
$5.5 \mathrm{~kb}$ fragment. Alignment of these sequences with that of SVA cDNA (4) supports the existence of four exons and three introns in the SVA gene ( $\mathrm{Fig}$. 2A). The $2.7 \mathrm{~kb}$ fragment covers the $5^{\prime}$-flanking region up to $-2135 \mathrm{bp}$ from the transcription initiation site, the first exon and one part of the first intron (462 $\mathrm{bp})$. The partial sequence of the $5.5 \mathrm{~kb}$ fragment extends from the other part of the first intron to the $3^{\prime}$ flanking region of $185 \mathrm{bp}$. Subcl oning and sequencing of a DNA fragment comprising the whole first intron amplified by polymerase chain reaction using the lambda 11S DNA as the template confirmed the linkage of Sacl site between the 2.7 and $5.5 \mathrm{~kb}$ fragments in the first intron (Fig. 2A).

The established genomic sequence of SVA gene is shown in Fig. 2B. The four exons of 123, 136, 112 and 227 bp are separated by three introns of 1555, 1931 and $316 \mathrm{bp}$. All of the exon/intron boundaries conform to the GT-AG consensus rule. A noncanonical TATA box appears at 31 bp upstream of the transcription initiation site and no CAAT box element is present within the -100 bp of $5^{\prime}$-flanking region. Repeats of nucleotides with purine and pyrimidine bases are present around -800 to $-600 \mathrm{bp}$ and -1350 to $-1100 \mathrm{bp}$ regions, respectively.

Functional AREs have been identified at several regions of the andron-responsive genes. Many of them are within -200 to -100 bp of the $5^{\prime}$-flanking region of the corresponding genes such as those encoding for the prostate-specific antigen (7), human glandular kallikrien-1 (8), rat probasin (9) and mouse vas deferens protein (10). However, functional AREs at different locations of the androgen-responsive genes have been identified also. For instance, the AREs of the genes of mouse sex-limited protein (SIp) (11) and tyrosine aminotransferase (12) locate respectively in the regions around 1.9 and $2.5 \mathrm{~kb}$ upstream of the transcription initiation sites, and the genes of prostatein C3 (13) and rat $20 \mathrm{kDa}$ protein (14) have functional AREs in their first introns. Ten ARE-like sequences were identified in SVA gene from selection for the half-site of the consensus ARE palindrome, AGAACAnnnTGTTCT (Table 1). These potential AREs spread at several regions of SVA gene. Two sets of adjacent ARE-like sequences, one includes -213 to $-199 \mathrm{bp}$ and -124 to $-110 \mathrm{bp}$ in the $5^{\prime}$-flanking region and the other includes 2532 to 2546 bp and 2582 to 2596 bp in the second intron, are noticeable to have high degree of homology with the ARE consensus sequence. Further studies of nuclear protein-DNA binding analyses and the assays of reporter gene fusion plasmid are necessary to determine which one of these ARE-like sequences participates in the androgen-mediating transcription activity of SVA gene.

Many lines of evidence indicate the necessity of the cooperative interaction between hormone response 
A
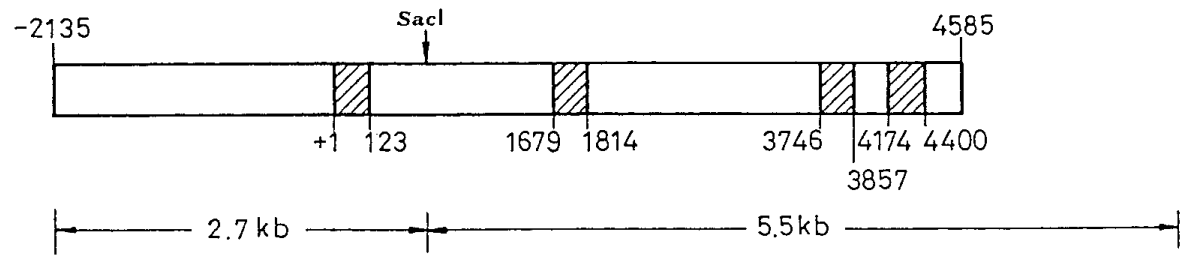

B

GAATTCTTAATTCTGAACATCTATGCTCCAAATGCAAAGGCCCCCATATTCATAAACTTTACTAA -2070 AGCTCAAAGCCTACAT TGCACCATAGACAATAACA.GTGAGAGACTTGACTTAAACACCACACTCTTAGCAATGAACAGTTCATGGAAACA - 1980 GA.A.CTAAGCAGAGACA.CAGTGAAACTTAACCGAAGTGGTGGACCAAGTGGATTTAA.CAGATATCTACAGAACATTTCATCCTAAAA.CAA -1890 AAGAATATACCTTCTTCTCAGCATCTCACTGTACCTTCTCCAAAACT GATTATATAATAGGTCACAAAACAGGCCTCAACAGATACARGA - 1800 AGATTGAAACAATCCCATGCATCCTATCAGAGTCACCACAGACTAAAGCTGCTCTICAATAACAATATAAACAACAGAAAGCCCACATAC - 1710 ACATGGAAGCTGAACAATGCCCTACTCAGT GATAACT TGGTCAAGGAAGAAATAAATAAAGAAATTAAAGACTAT TTTTGATTTAATTAA - 1620 CATGAAGGCCCCAGCATACCCAAACTTATGGGACACAATAAAAGCAGTGCTAAGAGGAAAATTCATAGCTCAGCTGCAAAAGAAACTGGA - 1530 A.GA.GCATACTATAGCAGCTTAACAGCATACCTGAAAGCTCTAGAACANAGAGAAGCAAATACACCCARTAGGAGTAGATGGCAGGAAAT - 1440 AAACTCAGGGCTGAAATCAACCAAGTAGAAACAAAAGGTACTANACAÄA.GAATCARCAAAACCAGGACCTGGTTCTTTGGTTCTTTGAGA -1350 AAATCTTCCTTCCTTTCTTCCTTTCTTCCTTTCTTCCTTTCTTCCTTTCTTCCTTTCTTCCTTTCTTCCTTTCTTCCTTTCTTCCTTCCT - 1260 TCCTTCCTTCCTCCCTCCAGTACATAGCCATCCATCCATCCATCCTTCCTTCCTTCCTTCCTTCCTTCTTTCCTCCCTCCTTCCCTCTCT - 1170 CCTTCCTCTCTCTCTCTCTCTCTCTCTCTCTCTCTCTCTCTCTCTCTCTCTCTCTCTCTCTTGTCCTTTTTTGTTTTTTATCAAATACAC - 1080 TCTAACAGGAGTCAAACTGAAAAAATAAACTTCAATTGAAAAAAGGTTTCCATCATATTGTCTCGTAGGCAAAAGATCATTGAGTATTTT -990 CTTGACTGATGATTTACTRTTGCAATCGCCATCCTGAACAGCTGGAATAAGAAATCGGTCTGCACAAGATGATTATTCTATTTCTACATG

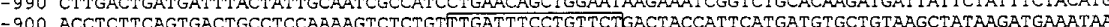
- 10 ACTCICOAG -720 AAGGAAGGA.GGAGGGGGGAGGAGG

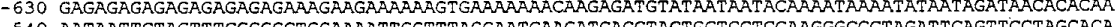
-540 ARTARTTCTAGTT

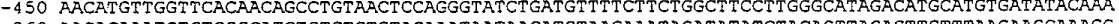
-360 AAGACAAATGTGTGGGCATGTGTGIGTGTACAAATAATAACATGTAAGAAATAGATATATCIACAGTTAGAGTTGTTTAAGAAGCAAAGG -270 ACAAAAAGATCAGATCTTCTTTAGAAAAATTGGAACTGGATCCATTCTAATAATCT'TAGAACATTCTAATCTCTTATGGAGAAATCTGAT

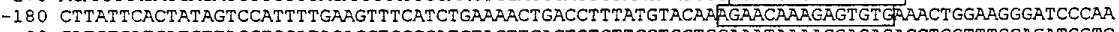
-90 CATCTGATCATCTTAGCTGGATAGACCTGCCCATCTACTTCACTGTCTTCCTGCTGCAAATAARAGGAGAGACCTGGTTTGCAGATGGTC +1 AAGGCTCATGTATGCCAACCCATACAGCATGGCTTCTCTTCAAAAGTTCTATCAACCTGGAGCAGTCACATATCTCCTTATTGTATTCTT MetAlaSerLeuGlnLYs PheTYrGlnProGlyAlaValThrTyrLeuteurleVal Phele
-20

91 ACTTCAGGAGACTGCTGGGTTTTGGTACCATGGGTAAGGCACTACTTTTTCCTCAGCATCTTCCTAGAAATATTTTTACACTTRACA.CAG ULEUGInGIUThrAlaGlyPhETRPTYxHISGI

181 AAACATAATGGATTTGTGCTGTATGTCAATCTGACTTTCTCTTTTCTAAACTCTGTGATGATGTGTCTTCTCAAACCTACATCCTGAAAT 181 AAACATAATGGATTTGTGCTGTATGTCAATCTGACTTTCTCTTTTCTAAACTCTGTGATGATGTGTCTTCTCAAACCTACATCCTGAAAT 271 CAAATGACTGGTGACAAAAGATTGTGTTCAAACTTACTTTTCTTCCTAGAT TATAGTATCATCACCATGCAATATGAAGATACAAGTGAG 361 CTTCGCTTATATTTTAGGATAAACTATGGACAGGATATAATTTATCTCTAAGGCTAAACTTGG TGTGGAMGCARATGATGGCAT GTAMATT 451 GAGTTCCCTCATGATAGAATAAAACATCATGTCACAACTAACCAAAACACAATGAATTAATTAGATATCTACAGGA.AAATTATCTAAATT 541 GTTTAATAAAACCTGATATCTTCATTAATTCTAATATGCA.GAGCTCTGTTAACCATACATGGAACA.GATTGGTTTAATAAAAAGAGAAGC
631 CGGGCGTAGTGGCACACGCCTTTARTCCAGCACTCAGGAGGCAGAGGCAGGCGGATTTCTGAGTTCCAGGCCAGCCTGGTGTACAAAGTG 721 AGTTCCAGGACAGCCAGGGCTGCA.CAGAGAAA.CCCTGTCTCAAAAGAGAGAGAGAGAGAGAGAGAGAGAGAGAGAGAGAGAGAGAGAGAG 811 AGAGAGAATGTCAGAACTTGATGCTGTACCCCAGCCTTTCTTTACTTGTTCCTTTACACAAGATTCCTTGAATAATAARTTTCTTTTCTA. 901 AATCTATAACTAAAGGGGGGAAATGTCTGATTTCAATTCCTATAAAATGAAAATAATATGTAAAGGGTCCTPAGACTTCTGACAAGAMGA 991 ACATGTTAAGTTACAGGAAGAAATCAGAATCTACTAACAATACCTAARATCAATAAGGAAAGGGGACTCTTCAAGATGGTAATGAATCCA 1081 TGGATATTAGTCTGGAAAATTTTTATGTGCCAAGTAAATAATTTTCCATAATTGAAGCAATGGACTGAGCAACTTAGGAAATGATCTTAA. 1171 GGTAATTTGAGTTGGAAATCAAATTTTTTCAACTAGAACTTTGATGCTTCCTATTTCAGACCTAAAGGGCAGTCAGATGAGGAAAAAATA 1261 TTGAGCTGAATCTGCAGGTACCATGAGACCAGGTTGACATTTATAATGAATTTAGCACTTTTGTGGCTCAATATATTTATRCAAGTCCTA 1351 CTTTTAGAAGGAAGAGAGTTGTGAAGACTTTTTTTGATACTTGTAAAAGAAACATAGGAAGGGGAGTAAGCAAAGATGGACGATGAAGTC 1441 ATAAATCTCCACTGGGAATGTTGCCTGGGAAACAAAAGCAACTTAACCAGCCCAGGACCCCACAATCTCCAGGTGACCAGGACTGC 1531 TACTCATTGTCCTGATTTCCCTTTGCTTCATCCTCCTGGAAGTGTCTGTTGAETAGAAATATGTTCTAACTTTGTGTGTGTGTGCTCTCT

1621 CTGTCTTCCCATACGAAAACAACTAAGTCTTAGGCAGTAATGTGAGTTTCTGTTTCAGCTTACACAATGAACCCCGTAACTACACACTCA YLEUHI SASnGLUPROARGASnTYTThrLeuT

1711 CACTGAATATGAAAATCACTCCAAGTACTAGCATTACTAACAAAAAAGGGATGAATTTCGAGTACAACTGACAGTTAAAAACAACGTGA

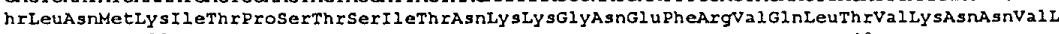
20 40

1801 AGCAATGCCAGGAGGTAAGCGAATAATTAAAAGTTAACATGAAAACTAAAATATTATATTTAAGATAACTCAATAATTAAATATTGTGC YSGInCYSGIIGLU

FIG. 2. Nucleotide sequence of the SVA gene. (A) Schematic diagram of the SVA gene. Cross-hatched bars are exons. Open bars are 5 '-flanking, introns and $3^{\prime}$-flanking regions. Nucleotide numbers are relative to the first transcription initiation site. (B) The established nucleotide sequence of the SVA gene. The two successive transcription initiation sites are marked by "*" and the first one is numbered +1 . Exon regions are typed in bold and coding regions are displayed with deduced amino acid sequences which are numbered according to the mature protein (4). The noncanonical TATA box at position $-31 \mathrm{bp}$ is double-underlined. The translation start, stop codons, and two overlapping polyadenylation signals are underlined with solid lines. The consensus sequences between the exon/intron boundaries are underlined with dashed lines. ARE-like sequences are boxed.

elements and other accessory response elements for the strong response and specificity of hormone action (15). Some important accessory response elements associated with the androgenic action have been found. A nuclear factor I (NF-I) binding site in the mouse mammary tumour virus long terminal repeat (16) and the binding sites of the octamer transcription factor 1 and the N F-I-like factor in the prostatein 


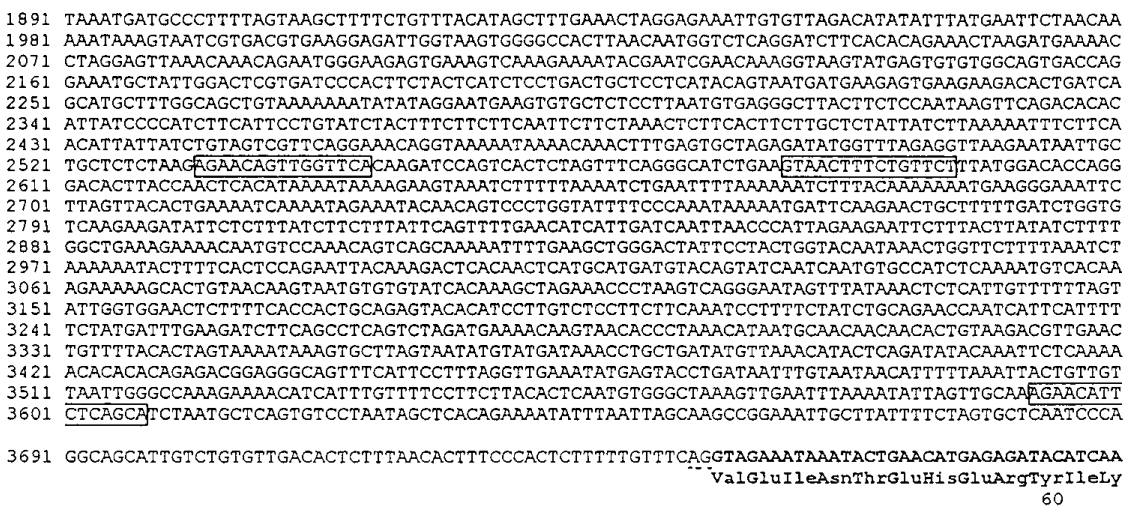

3781 GTTCCTTTCTGGTCCATCAACTTTTGAAACTTGCATATGTACCATTAGCGATTTCTTCTGGGATATATATGTCTCTGGTAAGTGGGAAAT sPheleuSerGlyProSerThrPheGluThxCYsI leCYsThrI leSerAspPhePheTrpAspIleTyrValserG

80

3871 TTTTCTACCTTCTTATGAAATTTTCAGATCAGATAACAGTAAATGTCAGAAGTATCAGAGTGGGTARAAAAATACATAAAACTACATTGT 3961 AGCTGGGTGAAAAGAGGGCAGGGGAGTCGAATATAGTACTATAAGAAACCAAATCCAAGTAGGGGAGAGAAGGAGCTGAAAGAAAGGAAA 4051 GAGAGTTCAAAGAGCAGCTGTACCTTATATATAGGAAAGGAATCAGTGTATTCTAARGTCAGCATGGGCTATGAATGGAGTTATATGGAG

4141 TTTATAACAACATTTCTACTTGTCTTTTTGTAGAAAGCACTTACCTAACACTATCAGCCTCCATTCTGCCATATAAGAACAAATGTCCTG

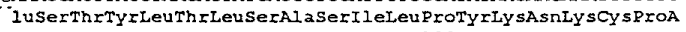
100

4231 ACTCTGACGAACCACTACTATATGGCGACTCTTACCAAATGTACAATATTACAGATAAAATCAATGTAACACCATAAACTGGAAGGTTGC

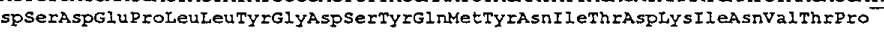

4321 AATAGAACCACATCACTAACGTACTCTTGGAGAGGATTGTGTGTGTGCGCATGTATTTTGCAATAAAATAAAATAGTGTTAAATTAATTC

4411 TGTGGTAAAATCTCTGTATGGTARATAAATTTTTTCCACCTCACAATTCACTATCTACATCCTTTAACCCAAAAGTATATCAGTAACGTA 4501 GAGAGAAAGAGAGAGAGTGTGTGTGTGTGTGTGTGAGAGAGAGGGGGAGTTTCATTCCTTTAGGTTGAAATATGA.GTATCTGATA

FIG. 2-Continued

C3 gene (17) were demonstrated to modulate the androgen response of the promoters. Accessory elements or other transcription factor binding sites contributing to the regulation of the androgen response are also believed to be present in the genes of the SIp (11) and rat 20-kDa protein (14). The cooperative interaction between ARE and accessory response elements might ensure the expression of androgen-dependent genes in specific tissues. The androgen-stimulated SVA expresses exclusively in the seminal vesicle (4) despite that the androgen receptor exists in many other sexual and non-sexual organs. Regula- tion of the transcription of the SVA gene might be dependent on a specific machinery including a novel transcription factor(s) in seminal vesicle. Several potential binding sites for various transcription factors were identified in the SVA gene. Within the 5' -flanking region, there are potential binding sites for CACCC-binding protein (position -1468) (18), roddent PitB 1 and PitB2 factor (position -582) (19) and GATA factor (position -532) (20). Whether these factors or other elements in the SVA gene participate in the control of the specific expression of SVA awaits future study.

TABLE 1

The ARE-like Sequences in SVA Gene

\begin{tabular}{|c|c|c|c|}
\hline Position & Nucleotide no. & Sequence & Homology \\
\hline ARE consensus & & AGAACA nnn TGTTCT & $100 \%$ \\
\hline \multirow[t]{5}{*}{ 5'-Flanking } & $-1912 \sim-1898$ & AGAACA ttt caTcCT & $75 \%$ \\
\hline & $-1488 \sim-1474$ & AGAACA aag aGaagc & $58 \%$ \\
\hline & $-867 \sim-853$ & ttgAtt tCC TGTTCT & $58 \%$ \\
\hline & $-213 \sim-199$ & AGAACA ttc TaaTCT & $83 \%$ \\
\hline & $-124 \sim-110$ & AGAACA aag aGTgTg & $75 \%$ \\
\hline \multirow[t]{2}{*}{ First intron } & $988 \sim 1002$ & AGAACA tgt TaagtT & $67 \%$ \\
\hline & $1583 \sim 1597$ & ctAgaA ata TGTTCT & $67 \%$ \\
\hline \multirow[t]{3}{*}{ Second intron } & $2532 \sim 2546$ & AGAACA gtt gGTTCa & $83 \%$ \\
\hline & $2582 \sim 2596$ & gtAACt ttc TGTTCT & $75 \%$ \\
\hline & $3593 \sim 3607$ & AGAACA ttc TcagCa & $67 \%$ \\
\hline
\end{tabular}




\section{ACKNOWLEDGMENT}

This work was partially supported by Grant NSC-86-2311-B-001081 from National Science Council, Taiwan.

\section{REFERENCES}

1. Ostrowski, M. C., Kistler, M. K., and Kistler, W. S. (1979) J . Biol . Chem. 254, 383- 390.

2. Mansson, P. E., Sugino, A., and Harris, S. E. (1981) Nucl. Acids Res. 9, 935-946.

3. Chen, Y. H., Pentecost, B. T., Mclachlan, J . A., and Teng, C. T. (1987) Mol. Endocrinol. 1, 707-716.

4. Yu, L.-C., Chen, J .-L., Tsai, W.-B., and Chen, Y.-H. (1993) Biochem. J. 296, 571- 576.

5. Huang, Y.-H., Luo, C.-W., Yu, L.-C., Chu, S.-K., and Chen, Y.-H. (1995) Biophys. J . 69, 2084- 2089.

6. Zhou, Z.-X., Wong, C.-I., Sar, M., and Wilson, E. M. (1994) Recent Prog. Horm. Res. 49, 249- 274.

7. Riegman, P. H.J ., Vlietstra, R. J ., van der Korput, J . A. G. M., Brinkmann, A. O., and Trapman, J . (1991) Mol. Endocrinol. 5, 1921- 1930.

8. Murtha, P., Tindall, D. J ., and Young, C. Y. F . (1993) Biochemistry 32, 6459-6464.
9. Rennie, P.S., Bruchovsky, N., Leco, K.J ., Sheppard, P. C., McQueen, S. A., Cheng, H., Snoek, R., Hamel, A., Bock, M. E., MacDonald, B.S., Nickel, B.E., Chang, C., Liao, S., Cattini, P. A., and Matusik, R. J . (1993) Mol. Endocrinol. 7, 23-36.

10. Fabre, S., Manin, M., Pailhoux, E., Veyssière, G., and J ean, C. (1994) J . Biol. Chem. 269, 5857- 5864.

11. Alder, A. J ., Scheller, A., Hoffman, Y., and Robins, D. M. (1991) Mol. Endocrinol. 5, 1587- 1596.

12. Denison, S. H., Sands, A., and Tindall, D. J . (1989) Endocrinology 124, 1091- 1093.

13. Tan, J., Marschke, K. B., Ho, K.-C., Perry, S. T., Wilson, E. M., and French, F. S. (1992) J . Biol. Chem. 267, 4456- 4466.

14. Ho, K.-C., Marschke, K.B., Tan, J., Power, S. G. A., Wilson, E. M., French, F. S. (1993) J . Biol. Chem. 268, 27226- 27235.

15. Lucas, P. C., and Granner, D. K. (1992) Annu. Rev. Biochem. 61 , $1131-1173$.

16. Cato, A. C. B., Skroch, P., Weinmann, J ., Butkeraitis, P., and Ponta, H. (1988) EMBO J . 7, 1403-1410.

17. Celis, L., Claessens, F., Peeters, B., Heyns, W., Verhoeven, G., and Rombauts, W. (1993) Mol. Cellular Endocrinol. 94, 165172.

18. Schule, R., Muller, M., Otsuka-Murakami, H., and Reinkawit, R. (1988) Nature 332, 87-90.

19. Chen, R., Ingraham, H. A., Treacy, M. A., Albert, V. R., Wilson, L., and Rosenfeld, M. G. (1990) Nature 346, 583- 586.

20. Orkin, S. H. (1990) Cell 63, 665-672. 